performing MCI. Further prospective studies are warranted to evaluate if the MCI technique affects reperfusion and functional outcomes in endovascular patients.

Disclosures M. Nouri: None. J. Lee: None. J. Mocco: None. P. Singh: 6; C; Penumbra.

\section{E-138 ENDOVASCULAR MANAGEMENT OF FREE FLOATING COMMON CAROTID THROMBI}

${ }^{1} \mathrm{~K}$ Carr ${ }^{*},{ }^{2} Z$ Thwing, ${ }^{2} \mathrm{R}$ Shivashakar, ${ }^{2} Y$ Serulle. ${ }^{1}$ Aventura Hospital and Medical Center, Coral Springs, FL; ${ }^{2}$ Aventura Hospital and Medical Center, Aventura, FL

\subsection{6/neurintsurg-2019-SNIS.213}

Background Free floating thrombi of the common carotid artery are rare causes of acute stroke syndromes. In previously described cases, authors have either advocated surgical management or conservative anticoagulation therapy. We described two cases in which endovascular management provided definitive and longstanding treatment.

Case 1 A 51-year-old Hispanic woman with a history of insulin dependent diabetes mellitus (IDDM), hypertension and hyperlipidemia presented with 2-3 days of waxing and waning right sided hemiparesis, aphasia and paresthesia. Initial noncontrast-enhanced head computed tomography (NECT) examination demonstrated no evidence of acute intracranial pathology. Doppler sonogram and cervical angiogram confirmed the presence of the thrombus with $70-80 \%$ stenosis. CT angiogram of the brain vasculature demonstrated no evidence of intracranial large vessel occlusion. Brain Magnetic Resonance Imaging (MRI) demonstrated acute watershed infarcts in the left cerebral hemisphere as well as a few punctate embolic infarcts. Serologic workup was significant only for hyperglycemia. After a brief course of heparinization, successful mechanical revascularization was accomplished using direct aspiration of the thrombus.

Case 2 A 51-year-old African American male with a history of insulin dependent diabetes mellitus and hyperlipidemia presented emergently after 6 hours of acute aphasia and right sided hemiparesis. Initial NECT demonstrated left middle cerebral artery (MCA) territory infarcts involving the frontal and temporal lobes. Cervical CT Angiogram demonstrated a long segment free floating thrombus of the distal left common carotid artery (CCA), which was confirmed on subsequent cervical angiogram. In this case, mechanical revascularization was accomplished with aspiration and stentretriever, followed by balloon angioplasty.

Conclusion Endovascular mechanical revascularization is an efficient and definitive treatment in patients with free floating carotid thrombi.

Disclosures K. Carr: None. Z. Thwing: None. R. Shivashakar: None. Y. Serulle: None.

\section{E-139 THE CHARACTERISTICS OF ELDERLY PATIENTS OVER 80 YEARS OF AGE WHO UNDERWENT MECHANICAL THROMBECTOMY USING STENT-RETRIEVERS DUE TO ACUTE CEREBRAL INFARCTION}

K Jang*, B Cho, B Moon, K Kim, D Jang. Neurosurgery, Incheon St. Mary's hospital, The Catholic University of Korea, Bupyeong-gu, Incheon, Korea, republic of

10.1136/neurintsurg-2019-SNIS.214
Introduction Recently, the average life expectancy of people has increased, and the number of cerebral infarctions in elderly patients over 80 years of age is also increasing. There are increasing cases of mechanical thrombectomy using stent-retriever in elderly patient, but the characteristics and differences of the patient population are not well established yet. The purpose of this study is to investigate the characteristics and prognostic factors of elderly patients over 80 years of age undergoing stent-retriever thrombectomy.

Methods From 2013 to 2017, we analyzed the prospective gathered data of 190 consecutive patients treated with mechanical thrombectomy using stent-retrievers for acute ischemic stroke in a single center. Demographic, clinical, laboratory, and radiologic features of each patient were invasgated. We compared the characteristics of elderly patients over 80 year-old with those of younger patients and evaluated the prognostic factors associated with good outcomes in elderly patients.

Results Of the total 133 patients, 34 patients (25.6\%) were over 80 years old. Initial ASPECT score (9.41 vs 8.78 , $\mathrm{p}=0.016), 30$ days $\mathrm{mRS}(4,21$ vs $3.18, \mathrm{p}=0.003)$ and 90 days $\mathrm{mRS}(4.15$ vs $2.95, \mathrm{p}=0.001)$ were significantly higher at elderly patients. Hypertension history $(p<0.001)$, underlying atrial fibrillation $(p=0.007)$ were significantly more common in elderly patients. The favorable outcomes $(m R S \leq 2, p=0.035)$ were significantly different between the two groups. Because the 30 days and 90days mRS were higher in the elderly patients after mechanical thrombectomy, prognosis may be worse and other complications should be noted.

Conclusions Elderly patients over 80 year-old had a few more underlying diseases, and the functional outcome was slightly worse after mechanical thrombectomy using stent retriever in acute cerebral infarction. However, the prognostic factors such as mortality and favorable outcome did not differ significantly between the two groups. Elderly patients should also consider aggressive treatment while appropriately controlling underlying diseases.

Disclosures K. Jang: None. B. Cho: None. B. Moon: None. K. Kim: None. D. Jang: None.

\section{E-140 STRATEGIZING TANDEM OCCLUSION ACUTE STROKE MANAGEMENT BASED ON CTA}

'S Paramasivam*, ${ }^{2} S$ Kumar. 'Neurosurgery, Apollo Hospitals, Chennai, India; ${ }^{2}$ Neurosurgery, Apollo Hospitals, Bangalore, India

10.1136/neurintsurg-2019-SNIS.215

Objectives To strategize the management of major stroke secondary to tandem occlusions of extracranial ICA and Intracranial ICA/MCA based on the collateral assessment in the preprocedure CTA.

Methods In a retrospective analysis of our Stroke Thrombectomy database $(\mathrm{n}=85)$, treated between August 2016 to December 2018 we identified 15 patients who presented with tandem occlusion. 13 were atherosclerotic disease and 2 were carotid dissection along with middle cerebral artery or distal ICA occlusion. All patients had pre-procedure CT angiogram, along with the primary pathology, collaterals comprising of Acom and Pcom arteries and their caliber was analyzed. All except 3 patients were treated with balloon 


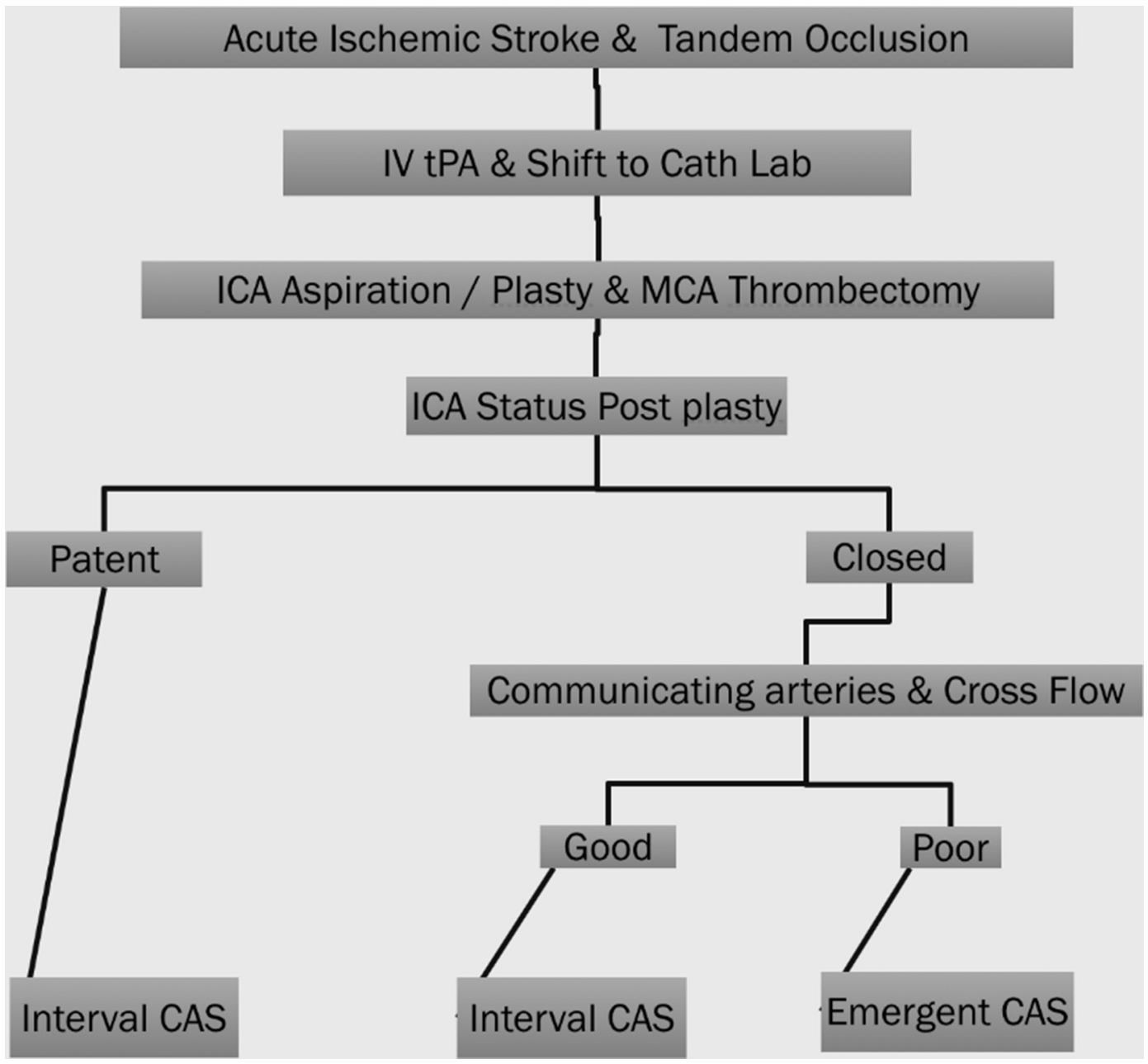

\section{Abstract E-140 Figure 1}

angioplasty. 4 patients had carotid stenting. The decision on emergent stenting was based on the algorithm shown in Image attachment. Stenting is done following Bolus GP2B3A inhibitor followed by infusion for 6 hours and Asprin and Plavix given through NG tube. Successful recanalization based on thrombolysis in cerebral infarction (TICI) score of $2 b$ or 3 and neurological improvement is defined by $\geq 8$ point reduction of National Institutes of Health Stroke Scale (NIHSS) score at 7 days and an improved modified Rankin Scale $(\mathrm{mRS} \leq 2)$ score at 90 days.

Results Overall, 93\% had TICI 2b/3 signifying successful recanalization. $46.6 \%$ had a reduction in the NIHSS score by $\geq 8$ points at 1 week. $60 \%$ had a good outcome with a $\mathrm{mRS} \leq 2$. Mortality was $12.5 \%(\mathrm{n}=2)$ with 1 deaths due to massive hemorrhage and 1 death due to cardiac cause.

Conclusions Major Strokes due to tandem occlusion of ICA and ICA/MCA can be treated successfully with lesser morbidity and mortality if strategized based on the understanding of collateral circulation based on pre-procedure CT angiogram. The number of stenting can be minimized and reserved for only those patients with a poor flow in the proximal ICA and poor collateral circulation and those with carotid dissection.

Disclosures S. Paramasivam: None. S. Kumar: None.

\section{E-141 LARGE VESSEL OCCLUSION STROKE THROMBECTOMY IN THE ELDERLY: IS 90 THE NEW 80 ?}

M Bouslama*, G Rodrigues, L Pisani, D Haussen, M Frankel, R Nogueira. Emory University/ Grady Memorial Hospital, Atlanta, GA

\subsection{6/neurintsurg-2019-SNIS.216}

Background Little is known on the outcomes of nonagenarians treated with endovascular therapy (ET) for acute large vessel occlusion stroke. It remains unclear whether they have worse clinical outcomes than octogenarians.

Methods We reviewed our prospectively collected endovascular database at a tertiary care academic institution between 09/ 2010-11/2018. All patients older than 80 years that underwent endovascular therapy for large vessel occlusion anterior circulation acute ischemic stroke were included and categorized into two groups: 80-89 years (octogenarians), and 9099 years(nonagenarians). Baseline, procedural, and radiological characteristics, as well as outcome parameters were compared. Receiver operating characteristic curves were used to calculate the optimal final infarct volume (FIV) threshold to predict good outcomes in both age category.

Results Fifty nonagenarians and 248 octogenarians were treated over the 8-year study period. When compared with octogenarians, nonagenarians were less often males $(18 \%$ vs 